\title{
Compensação ambiental: análise legal da reparação financeira dos impactos ambientais
}

\section{Ragner Kennedy Silva Pessoa*, Thiego Henrique Siqueira Moura, Werner Farkatt Tabosa}

Universidade Potiguar (UNP). Av. Senador Salgado Filho, 1610. Lagoa Nova. Natal-RN. Brasil. (CEP 59056-000).*E-mail: ragnerkennedy@gmail.com.

Resumo: A compensação ambiental stricto sensu é um dispositivo de lei com a finalidade de reparar financeiramente impactos ambientais nos empreendimentos licenciados através do Estudo de Impacto Ambiental e respectivo Relatório de Impacto Ambiental (EIA/RIMA). Expresso no art. 36, da Lei n ${ }^{\circ}$ 9.985/2000, a Lei do Sistema Nacional de Unidades de Conservação (SNUC), e posteriormente regulamentado pelo Decreto $\mathrm{n}^{\circ} 4.340 / 2002$, o dispositivo tem prioridades definidas para a aplicação dos valores arrecadados que em sua maioria sustentam a implantação e manutenção das unidades de conservação. O Decreto n ${ }^{\circ}$ 6.848/2009 define um teto de $0,5 \%$ (meio por cento) dos custos totais para implantação do empreendimento e cria as fórmulas para o cálculo da compensação ambiental, entretanto, este mecanismo apresenta fragilidades e necessita de formas para fortalecimento do mesmo, diante disso foram aplicados testes estatísticos com o objetivo de refutar algumas das diretrizes e procedimentos da cobrança por compensação ambiental, através de análises quantitativas e qualitativas, consultas aos sites de órgãos federais, como o Ministério do Meio Ambiente e o Palácio do Planalto, compreendendo seus fundamentos legais e metodológicos. As planilhas na plataforma Office-Excel elaboradas baseando-se no decreto, foram preenchidas com dados aleatórios, apresentam alguns conflitos e incongruência que podem inviabilizar a cobrança da compensação ambiental, ou no mínimo confundir os profissionais que utilizam a metodologia, deste modo, é evidente que a compensação ambiental stricto sensu ainda necessita de ajustes, que possam tornar mais justa e aplicável, para cada vez mais fortalecer o processo de licenciamento ambiental.

Palavras-chave: Compensação ambiental; Licenciamento ambiental; EIA/RIMA; Unidades de conservação.

Abstract. Environmental compensation: an analysis of financial repair mechanism of the environment. The Environmental Compensation (EC) stricto sensu is an instrument of law that aims to compensate economically environmental impacts in enterprises licensed to EIA/RIMA. According to article 36, of Law No. 9.985/2000, the Unity Conservation National System (SNUC) Law, and afterwards through of the Decree No. 4.340/2002, the environmental compensation has priorities defined for application of values that predominantly implement and maintain Conservation Units. The Decree No. 6.848/2009 defines a ceiling of $0.5 \%$ (half
Recebido:

24/05/2017

Aceito:

29/06/2017

Publicado:

30/06/2017

Acesso Aberto Artigo completo

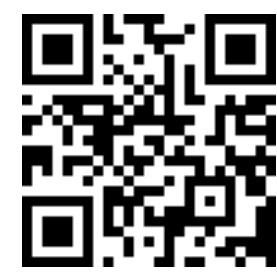

ORCIID

(1) 0000-0001-7425-9590

Ragner Kennedy Silva Pessoa

(D) $0000-0002-9387-4035$

Thiego Henrique

Siqueira Moura

(ㄷ) 0000-0002-6775-0215

Werner Farkatt Tabosa 
percent) of the total costs at the implementation of enterprises and creates formulas for calculating environmental compensation. However, that methodology has weaknesses and needs to be strengthened. Thus, we applied statistical tests to refute some guidelines and to cash in on Environment Compensation. In order to do it, we used qualitative and quantitative analysis, as well we researched into websites of Federal Agencies, for example, Ministry of the Environment and Palácio do Planalto, studying its legal and methodological principles. Ours spreadsheets developed in Excel Office, based on decree, were completed with random data and they have some conflicts and incongruities these not collaborate with the cash in on EC, or at least confuse professionals. In that sense, it is evident that environment compensation strict sensu still needs adjustments to make itself more equal and useful to solidify even more the environment licensing process.

Keywords: Environmental compensation Environmental licensing; EIA/RIMA; Conservation units.

\section{Introdução}

A Compensação Ambiental stricto sensu é um dispositivo de lei com a finalidade de reparar financeiramente impactos ambientais considerados não mitigáveis, constatado pelo órgão licenciador após análise do EIA/RIMA que é submetido para fim de concessão da licença ambiental. Expresso no art. 36, da Lei $\mathrm{n}^{0}$ 9.985/2000, a Lei do Sistema Nacional de Unidades de Conservação (SNUC), e posteriormente regulamentado pelo Decreto $n^{\circ} 4.340 / 2002$, o dispositivo tem prioridades definidas para a aplicação dos valores arrecadados que em sua maioria sustentam a implantação e manutenção de unidades de conservação, sejam elas de proteção integral ou de uso sustentável, sendo que esses recursos devem ser utilizados "principalmente em regularização fundiária, demarcação das terras, elaboração, revisão ou implantação do plano de manejo, aquisição de bens e serviços necessários à implantação, gestão, monitoramento e proteção da unidade, compreendendo sua área de amortecimento, desenvolvimento de estudos necessários à criação de nova unidade de conservação e desenvolvimento de pesquisas necessárias para o manejo da unidade de conservação e sua área de amortecimento”.
A concepção de reparação financeira dos danos e/ou impactos ambientais causados por empreendimentos que buscam licença ambiental tem fundamentos anteriores à Lei do SNUC. O Conselho Nacional do Meio Ambiente (CONAMA), na Resolução $n^{\circ}$ 10/1987, determina que, como modo de reparar os danos causados ao ecossistema, no ato do licenciamento, fundamentado no Relatório de Impacto Ambiental (RIMA), o empreendimento que for considerado pelo órgão licenciador como obra de grande porte, compensaria os danos ambientais com a implantação de uma Estação Ecológica e o total gasto na implantação e benfeitorias não poderia ser inferior a $0,5 \%$ (meio por cento) dos custos totais previstos para a implantação do empreendimento.

Em 1988, com a promulgação da Constituição Federal, a reparação financeira dos danos ambientais tem sua legalidade respaldada, quando no art. 225, $\S 3^{\circ}$, estabelece que condutas ou atividades consideradas lesivas ao meio ambiente caberiam de sanções penais e administrativas, independentemente da obrigação de reparar os danos causados.

Somente em 1996 o CONAMA editou a Resolução ${ }^{\circ}$ 2, e determinou que outras unidades de conservação, além de Estação Ecológica, também poderiam ser 
beneficiadas pelo montante arrecadado pela reparação financeira dos danos ambientais. Justificado a cobrança mínima de 0,5\% (meio por cento) dos custos totais para implantação do empreendimento quando o empreendimento fosse considerado pelo órgão ambiental competente como obra de relevante impacto ambiental fundamentado no então Estudo de Impacto Ambiental e respectivo Relatório de Impacto Ambiental (EIA/RIMA).

Em 2000, o art. 36, do SNUC, traz as fundamentações legais para que 0 empreendimento de significativo impacto ambiental, assim considerado pelo órgão ambiental competente, com fundamento em estudos ambientais realizados durante 0 processo de licenciamento ambiental, sendo considerados os impactos não mitigáveis e passíveis de riscos, apoiariam a implantação e manutenção de unidade de conservação do Grupo de Proteção Integral.

Após dois anos, o art. 31, do Decreto $\mathrm{n}^{\circ}$ 4.340/2002, regulamentou o SNUC e a compensação ambiental stricto sensu. E então se instituiu a compensação ambiental no sentido mais estreito do termo, tornando isto um dispositivo legítimo para reparar financeiramente os impactos não mitigáveis e passiveis de risco. Em 2005, com o Decreto $n^{0}$ 5.566, que altera o art. 31, do Decreto 4.340/2002 e dá nova redação ao caput, ratificando o EIA/RIMA como principal e exclusivo estudo ambiental para fundamentação da cobrança por reparação dos impactos ambientais não mitigáveis.

Uma Ação Direta de Inconstitucionalidade (ADIn) $\mathrm{n}^{0} 3.378 / 2004$, formulada pela Confederação Nacional da Indústria, contesta a cobrança por compensação ambiental e os parágrafos do art. 36, da Lei do SNUC. Durante todo o trâmite do processo que durou quatro anos, ainda em 2006, o CONAMA editou a Resolução $n^{\circ}$ 371 e no seu art. 15 fixou o pagamento da compensação ambiental em $0,5 \%$ (meio por cento) do valor do empreendimento até que o órgão ambiental estabelecesse metodologia para definir o grau de impacto.

Apenas em 2008 o STF julga a ADIn $n^{\circ} 3.378 / 2004$ e confirma a legali- dade da cobrança por compensação ambiental. Contudo, torna inconstitucional parte do texto do art. 36, da Lei $\mathrm{n}^{0} 9.985 / 2000$, onde diz que o valor cobrado: "não pode ser inferior a meio por cento dos custos totais previstos na implantação de empreendimento”. E que seria desnecessário a fixação do percentual pago sobre os custos do empreendimento.

Contudo, o Decreto $\mathrm{n}^{\circ}$ 6.848/2009 alterou o Decreto $n^{\circ} 4.340 / 2004$, definindo um teto de $0,5 \%$ (meio por cento) dos custos totais para implantação do empreendimento, cria as fórmulas para o cálculo da compensação ambiental (CA), onde o valor da CA é resultante do produto do Grau de Impacto (GI), com o Valor de Referência (VR), quando o valor de GI é obtido através da soma dos valores de Impacto sobre a Biodiversidade (ISB), Comprometimento da Área Prioritária (CAP) e a Influência sobre Unidade de Conservação (IUC) e o VR é o valor total gasto na implantação do empreendimento. Os valores de ISB, CAP e IUC são obtidos através de conversões dos índices dispostos em quadros no item 2 do anexo do decreto.

Diante disso, considera-se que 0 Ministério do Meio Ambiente cumpre a decisão do STF. No entanto, o Valor de Referência (VR), expresso na fórmula para o cálculo da compensação ambiental ainda está vinculado ao custo total do empreendimento, quando na verdade deveria ser necessário observar o valor dos significativos impactos ao ecossistema e aos serviços ambientais existente no local onde fora implantado o empreendimento. Tendo observado esta fragilidade do dispositivo de compensação ambiental e através da interpretação do Decreto $\mathrm{n}^{\circ}$ $6.848 / 2009$, foi realizado um teste de aplicabilidade das fórmulas apresentadas, com o objetivo de refutar algumas das diretrizes e procedimentos da cobrança por compensação ambiental e estimular possíveis alterações ou adequações que fortaleçam o processo de licenciamento ambiental, considerando a importância que o dispositivo tem na implantação e manutenção de unidades de conservação. 


\section{Material e métodos}

Esta é uma análise de caráter qualitativo e quantitativo, com a finalidade de verificar a aplicabilidade das fórmulas do dispositivo de compensação ambiental, através de consultas aos sites de órgãos federais como o Ministério do Meio Ambiente e Palácio do Planalto, compreendendo seus fundamentos legais e metodológicos.

Inicialmente, foi elaborada uma planilha na plataforma M. S. Office Excel, editada conforme a interpretação do Decreto $\mathrm{n}^{\circ}$ 6.848/2009, onde a compensação ambiental (CA) é calculada através do valor atribuído a cada índice do impacto avaliado, que é inserido em sua respectiva variável de entrada (Célula, M. S. Office Excel), que, por conseguinte, possibilita a resolução das fórmulas. No item 1.3 do anexo do decreto, a Influência em Unidades de Conservação (IUC) tem os valores variando entre $0,05 \%$ e $0,15 \%$, "sendo que os valores podem ser considerados cumulativamente até o valor máximo de $0,15 \%$ " quando o impacto tiver influência em:

"G1: parque (nacional, estadual e municipal), reserva biológica, estação ecológica, refúgio de vida silvestre e monumento natural $=0,15 \%$;

G2: florestas (nacionais e estaduais) e reserva de fauna $=0,10 \%$;

G3: reserva extrativista e reserva de desenvolvimento sustentável $=0,10 \%$;

G4: área de proteção ambiental, área de relevante interesse ecológico e reservas particulares do patrimônio natural $=0,10 \%$; e

G5: zonas de amortecimento de unidades de conservação = 0,05\%”.

O item 2 expressa os quadros que explicam a variação do valor de cada índice em função de seus atributos. Com isso, foi utilizada a variação de 0 a 3 para o Índice de Magnitude (IM), Índice de
Biodiversidade (IB) e Índice de Comprometimento de Áreas Prioritárias (ICAP), e a variação de 1 a 4 para o Índice de Abrangência (IA) e o Índice de Temporalidade (IT). A conversão dos índices em Impacto sobre a Biodiversidade (ISB) e Comprometimento da Área Prioritária (CAP) junto ao IUC, geram o valor do Grau de Impacto (GI), que é multiplicado pelo Valor de Referência (VR) resultando no valor final da CA.

$$
\begin{aligned}
& \mathrm{CA}=\mathrm{VR} \times \mathrm{GI} ; \\
& \mathrm{GI}=\mathrm{ISB}+\mathrm{CAP}+\mathrm{IUC} .
\end{aligned}
$$

ISB = IM x IB (IA+IT) /140;

CAP = IM x ICAP x IT / 70;

$\mathrm{IUC}=\mathrm{G} 1, \mathrm{G} 2, \mathrm{G} 3, \mathrm{G} 4$ e/ou G5.

IM = Índice Magnitude 0 a 3;

IB = Índice Biodiversidade 0 a 3;

IA = Índice Abrangência 1 a 4;

IT = Índice Temporalidade 1 a 4; ICAP = Índice Comprometimento de Área Prioritária 0 a 3.

Para esta análise utilizamos uma diretriz do decreto descrita no parágrafo quarto do art. 31, que se refere à compensação ambiental que "poderá incidir sobre cada trecho, naqueles empreendimentos em que for emitida a licença de instalação por trecho". Dessa forma, foi adotado um cenário onde a planilha avalia quatro trechos (Figura 1). É preciso ressaltar que, o processo de avaliação trata o VR "com base nos investimentos que causam impactos ambientais, relativos ao trecho". No entanto, cada trecho também pode ser interpretado como empreendimentos independentes, pois possuem todas as variáveis necessárias e o VR é tido como os custos totais para implantação do empreendimento. 

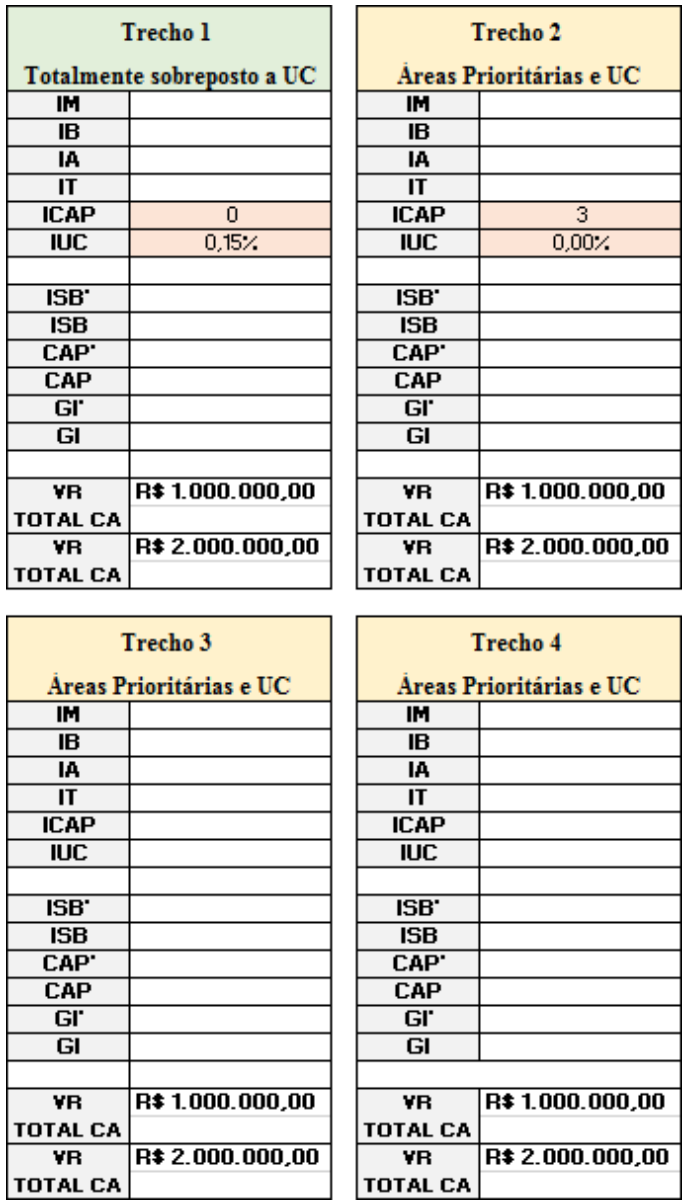

Figura 1. Quadros da planilha com os trechos do cenário avaliado.

O trecho 1 teve os impactos avaliados totalmente sobrepostos a unidades de conservação que, conforme o Decreto $\mathrm{n}^{\circ} 6.848 / 2009$ no item 2.5 do anexo, altera a fórmula final, tornando-se exclusivamente computado pelo IUC. Nessas condições o ICAP é zerado por se entender que não há impactos sobre áreas prioritárias ou os impactos em áreas prioritárias estão totalmente sobrepostos a unidades de conservação. Nos outros três trechos os impactos são avaliados em áreas prioritárias e unidades de conservação. Estes trechos, em sua maioria, tiveram os valores dos índices do impacto atribuído de maneira aleatória e conforme a variação dos atributos presentes nos quadros dos índices. Apenas 0 trecho 2 teve os índices totalmente iguais aos do trecho 1 e o ICAP fixado no valor máximo (3), assim como o trecho 1 teve o valor máximo atribuído ao IUC (Figura 2). Com isso, é possível verificar o peso real de cada índice no resultado do GI. E para que isso possa ser mais evidente o valor do IUC no trecho 2 também fica zerado, caso contrário, qualquer valor de IUC associado a ICAP teria maior peso no cálculo.

\begin{tabular}{|c|c|c|c|}
\hline \multicolumn{2}{|c|}{$\begin{array}{c}\text { Trecho l } \\
\text { Totalmente sobreposto a UC }\end{array}$} & \multicolumn{2}{|c|}{$\begin{array}{c}\text { Trecho } 2 \\
\text { Areas Prioritárias e UC }\end{array}$} \\
\hline IP & & $\| \mathbf{M}$ & \\
\hline IB & & IB & \\
\hline IA & & IA & \\
\hline IT & & IT & \\
\hline ICAP & 0 & ICAP & 3 \\
\hline IUC & $0,15 \%$ & IUC & $0,00 \%$ \\
\hline ISB" & & ISB' & \\
\hline ISB & & ISB & \\
\hline CAP & & CAP & \\
\hline CAP & & CAP & \\
\hline $\mathrm{GI}^{\circ}$ & & $\mathrm{GI}^{\circ}$ & \\
\hline GI & & GI & \\
\hline $\begin{array}{c}\text { VR } \\
\text { TOTAL CA }\end{array}$ & $R \$ 1.000 .000,00$ & $\begin{array}{c}\text { VR } \\
\text { TOTAL CA }\end{array}$ & $R \neq 1.000 .000,00$ \\
\hline $\begin{array}{c}\text { VR } \\
\text { TOTAL CA }\end{array}$ & $R \leqslant 2.000 .000,00$ & $\begin{array}{c}\text { VR } \\
\text { TOTAL CA }\end{array}$ & R\$2.000.000,00 \\
\hline
\end{tabular}

Figura 2. Fixação dos valores de ICAP e IUC.

Os índices são calculados conforme as fórmulas do decreto, resultando nos valores de ISB, CAP e, consequentemente, o GI. O Decreto $n^{\circ}$ 6.848/2009 determina que o ISB terá seu valor variando entre $0 \mathrm{e}$ $0,25 \%$, bem como o valor do CAP e o GI entre 0 e $0,5 \%$. Realizando um pequeno teste com as fórmulas é possível concluir que é comum ultrapassar esses tetos, devido a isto foi criado um item extra para cada resultado: ISB'; CAP'; GI'. Onde, o símbolo da aspa simples (') representa o item que não sofreu alteração no cálculo em função do teto estabelecido pelo decreto, ou seja, os resultados são transcritos de maneira real a que foram calculados (Figura 3). Os itens IBS, CAP e GI (sem aspas simples) são resultados ajustados conforme $\mathrm{o}$ teto estabelecido quando os valores reais são ultrapassados. 


\begin{tabular}{|c|c|}
\hline \multicolumn{2}{|c|}{ Trecho 3 } \\
\multicolumn{2}{|c|}{ Areas Prioritárias e UC } \\
\hline IM & \\
\hline IB & \\
\hline IA & \\
\hline IT & \\
\hline ICAP & \\
\hline IUC & \\
\hline & \\
\hline ISB' & \\
\hline ISB & \\
\hline CAP' & \\
\hline CAP & \\
\hline GI & \\
\hline GI & \\
\hline & \\
\hline VR & $\mathbf{R} \$ \mathbf{1 . 0 0 0 . 0 0 0 , 0 0}$ \\
TOTAL CA & \\
\hline VR & $\mathbf{R} \$ 2.000 .000,00$ \\
TOTAL CA & \\
\hline
\end{tabular}

\begin{tabular}{|c|l|}
\hline \multicolumn{2}{|c|}{ Trecho 4 } \\
\multicolumn{2}{|c|}{ Areas Prioritárias e UC } \\
\hline IM & \\
\hline IB & \\
\hline IA & \\
\hline IT & \\
\hline ICAP & \\
\hline IUC & \\
\hline ISB' & \\
\hline ISB & \\
\hline CAP* & \\
\hline CAP & \\
\hline GI & \\
\hline GI & \\
\hline VR & R\$ $\mathbf{1 . 0 0 0 . 0 0 0 , 0 0}$ \\
\hline TOTAL CA & \\
\hline VR & R $2.000 .000,00$ \\
TOTAL CA & \\
\hline
\end{tabular}

Figura 3. Itens dos cálculos reais e cálculos ajustados.

O GI de cada trecho foi multiplicado por dois Valores de Referência (VR) diferentes, R\$1.000.000,00 e $\mathrm{R} \$ 2.000 .000,00$, para que também fosse possível ter parâmetros comparativos entre os valores da CA em função dos custos de implantação dos empreendimentos (Figura 4).

\begin{tabular}{|c|c|}
\hline \multicolumn{2}{|c|}{ Trecho 3 } \\
Àreas Prioritárias e UC \\
\hline IH & \\
\hline IB & \\
\hline IA & \\
\hline IT & \\
\hline ICAP & \\
\hline IUC & \\
\hline & \\
\hline ISB' & \\
\hline ISB & \\
\hline CAP' & \\
\hline CAP & \\
\hline GI & \\
\hline GI & \\
\hline & \\
\hline VR & R $\$ \mathbf{1 . 0 0 0 . 0 0 0 . 0 0}$ \\
TOTAL CA & \\
\hline VR & R\$2.000.000.00 \\
TOTAL CA & \\
\hline
\end{tabular}

\begin{tabular}{|c|c|}
\hline & Trecho 4 \\
\hline Areas $\mathrm{P}$ & rioritárias e UC \\
\hline IH & \\
\hline IB & \\
\hline IA & \\
\hline IT & \\
\hline ICAP & \\
\hline IUC & \\
\hline ISB' & \\
\hline ISB & \\
\hline CAP' & \\
\hline CAP & \\
\hline $\mathrm{Gl}^{\circ}$ & \\
\hline GI & \\
\hline $\begin{array}{c}\text { YR } \\
\text { TOTAL CA }\end{array}$ & $R \$ 1.000 .000,00$ \\
\hline $\begin{array}{c}\text { YR } \\
\text { TOTAL CA }\end{array}$ & $R \leqslant 2.000 .000,00$ \\
\hline
\end{tabular}

Figura 4. Cálculo tem como base doisValores de Referência (VR) diferentes.

Por fim, os valores resultantes dos cálculos realizados pela planilha possibilitaram as análises e comparações, observando a coerência da metodologia do cálculo em função dos valores obtidos pela planilha nos resultados dos índices (ISB, CAP), os valores dos GI e o valor final de cada CA, bem como qualquer outro fato pertinente e que pudesse surgir durante a execução destas análises.

\section{Resultados e discussão}

Os cálculos realizados pela planilha apresentam alguns conflitos e incongruência que podem inviabilizar a cobrança da Compensação Ambiental, ou no mínimo confundir os profissionais que utilizam a metodologia. Com o completo preenchimento dos índices e a resolução das fórmulas podemos, a princípio, confirmar que os ajustes de ISB, CAP e GI são consideravelmente constantes tendo influência na redução do valor de GI (Figura 5).

O trecho1 que tem seus impactos totalmente sobrepostos a UC (ICAP Zerado) e que o IUC foi atribuído com valor máximo de $0,15 \%$, se comparado com o trecho 2 que os impactos são avaliados em Áreas Prioritárias com ICAP preenchido com valor máximo e IUC zerado, é possívelverificar que o peso real do IUC é inferior ao do ICAP tendo em vista que o IUC tem seu valor variando de $0,05 \%$ a $0,15 \%$ e o ICAP pode chegar a uma máxima de até $0,25 \%$, fazendo com que o CAP seja mais expressivo na fórmula final e impactos restritos a UC tenham menor influência no resultado do GI (Figura 5), em outras palavras, empreendimentos que venham a ser implantados impactando exclusivamente UC muitas vezes pagam menos na CA do que empreendimentos que tenham impactos fora de UC. Está é uma incongruência que eventualmente deverá sercolocada em discussão, caso contrário, as Unidades de Conservação já consolidadas estariam sendo descaracterizadas do papel de áreas protegidasmais restritivas. 


\begin{tabular}{|c|c|}
\hline & Trecho 1 \\
\hline Totalmen & te sobreposto a UC \\
\hline IM & 3 \\
\hline IB & 1 \\
\hline IA & 4 \\
\hline$\pi$ & 2 \\
\hline ICAP & 0 \\
\hline IUC & $0,15 \%$ \\
\hline ISB' & $0,13 \%$ \\
\hline ISB & $0,13 \%$ \\
\hline CAP' & $0,00 \%$ \\
\hline CAP & $0,00 \%$ \\
\hline $\mathbf{G l}^{\circ}$ & $0,28 \%$ \\
\hline GI & $0,28 \%$ \\
\hline VR & R\$ 1.000.000,00 \\
\hline TOTAL CA & $R \$ 2.785,71$ \\
\hline $\begin{array}{c}\text { VR } \\
\text { TOTAL CA }\end{array}$ & $\begin{array}{l}R \leqslant 2.000 .000,00 \\
R \leqslant 5.571,43\end{array}$ \\
\hline
\end{tabular}

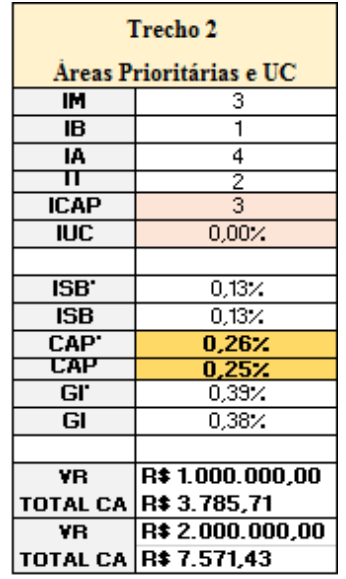

\begin{tabular}{|c|c|}
\hline \multirow{2}{*}{\multicolumn{2}{|c|}{$\begin{array}{c}\text { Trecho } 3 \\
\text { Areas Prioritárias e UC }\end{array}$}} \\
\hline & \\
\hline IM & 2 \\
\hline IB & 1 \\
\hline IA & 3 \\
\hline IT & 2 \\
\hline ICAP & 3 \\
\hline IUC & $0,05 \%$ \\
\hline ISB' & $0,07 \%$ \\
\hline ISB & $0,07 \%$ \\
\hline CAP' & $0,17 \%$ \\
\hline CAP & $0,17 \%$ \\
\hline GI & $0,29 \%$ \\
\hline GI & $0,29 \%$ \\
\hline $\begin{array}{c}\text { VR } \\
\text { TOTAL CA }\end{array}$ & $\begin{array}{l}R \$ 1.000 .000,00 \\
R \leqslant 2.928,57\end{array}$ \\
\hline $\begin{array}{c}\text { VR } \\
\text { TOTAL CA }\end{array}$ & $\begin{array}{l}R \leqslant 2.000 .000,00 \\
R \leqslant 5.857,14\end{array}$ \\
\hline
\end{tabular}

\begin{tabular}{|c|c|}
\hline \multirow{2}{*}{\multicolumn{2}{|c|}{$\begin{array}{c}\text { Trecho } 4 \\
\text { Areas Prioritárias e UC }\end{array}$}} \\
\hline & \\
\hline IM & 3 \\
\hline IB & 2 \\
\hline IA & 4 \\
\hline IT & 2 \\
\hline ICAP & 2 \\
\hline IUC & $0,10 \%$ \\
\hline ISB" & $0,26 \%$ \\
\hline ISB & $0,25 \%$ \\
\hline CAP' & $0,17 \%$ \\
\hline CAP & $0,17 \%$ \\
\hline $\mathbf{G I}^{\circ}$ & $0,53 \%$ \\
\hline GI & $0,50 \%$ \\
\hline $\begin{array}{c}\text { YR } \\
\text { TOTAL CA }\end{array}$ & $\begin{array}{l}R \leqslant 1.000 .000,00 \\
R \leqslant 5.000,00\end{array}$ \\
\hline $\begin{array}{c}\text { YR } \\
\text { TOTAL CA }\end{array}$ & $\begin{array}{l}R \leqslant 2.000 .000,00 \\
R \leqslant 10.000,00\end{array}$ \\
\hline
\end{tabular}

Figura 5. Reajustes de CAP, ISB e GI; Maior peso do CAP no trecho 2 por influência do ICAP em comparação ao IUC do trecho1.Comparação dos valores da CA de VR diferentes, tendo em vista a baixa relevância do GI e alta relevância no valor de implantação.

No caso dos trechos 3 e 4 é possível notar que, ter o valor de implantação do empreendimento como referência muitas vezes pode influenciar o resultado da CA de maneira equivocada, quando neste caso, o trecho 3 tem um valor de GI $(0,29 \%)$ razoavelmente mais abaixo que no trecho 4 $(0,50 \%)$, se comparado o valor da CA de VR de R\$ 2.000.000 do trecho 3 (R\$ $5.857,14)$ com o valor da CA de VR R\$ 1.000.000 do trecho 4 ( $\mathrm{R} \$ 5.000,00)$ é visível que o trecho 3 pagará uma CA superior ao trecho 4 apenas por possuir um valor de implantação maior, sem que haja muita relevância do GI apresentado (Figura 5). Isto mostra que, independente do GI, o empreendimento implantado sempre pagará sua CA em função do seu valor de implantação e que esta nunca será superior a $0,5 \%$ destes custos.O correto seria esperar que o empreendimento com maior Grau de Impacto tivesse o maior valor de CA, independentemente do seu valor de implantação, assim como o STF havia recomendado na decisão judicial da ADIn 3.378/2004, em 2008. Uma possível alternativa para este desacordo seria a Valoração Ambiental que por definição e conforme Mota (1997), é o valor econômico dos recursos ambientais sendo derivado de todos os seus atributos estando ou não associados a um uso. Muitas técnicas foram desenvolvidas para valorar economicamente o ambiente, em 2005 a Millennium Ecosystem Assessment apresenta categorias de serviços ambientais que podem ser valorados. Dentre as categorias existem vastos serviços associados à provisão, regulação, suporte e cultura. Como primeiro passo estes serviços devem ser identificados e definidos de acordo com as características do local e da população beneficiada pelos serviços prestados pelo ambiente. Uma metodologia que possa valorar economicamente $\mathrm{o}$ ambiente antes da licença prévia ser concedida ao empreendimento, que possa assumir o valor de referência na fórmula da Compensação Ambiental, aparenta ser mais justo tanto para o empreendedor quanto para o meio ambiente.

\section{Conclusões}

É evidente que a Compensação Ambiental stricto sensu ainda necessita de ajustes, que possa se tornar mais justa e aplicável, para cada vez mais fortalecer o processo de licenciamento ambiental que consequentemente proporciona maiores investimentos em Unidades de Conservação ajudando na manutenção e implantação de novas Unidades. O dispositivo deve ser aperfeiçoado e constantemente colocado em teste, para que as incongruências possam ser corrigidas com maior agilidade. É preciso considerar a possibilidade de uma capacitação para os profissionais que utilizam esta metodologia nos órgãos ambientais estaduais e municipais também, 
que possa ser feita através de eventos ou incentivos públicos. Ainda, é preciso que haja clareza sobre como o montante arrecado com CA está sendo aplicado e se todos os empreendimentos de grande impacto aptos a pagar CA estão contribuindo.

\section{Declaração de conflito de interesses}

Os autores declaram não haver conflito de interesses.

\section{Referências}

Brasil. Constituição da República Federativa do Brasil de 1988. Disponível em: $<$ http://www.planalto.gov.br/ccivil_03/constitui cao/constituicao.htm>. Acesso em: 03 mar. 2016.

Brasil. Lei $\mathbf{n}^{0}$ 9.985, de 18 de julho de 2000. Regulamenta o art. 225, § $1^{\circ}$, incisos I, II, III e VII da Constituição Federal, institui o Sistema Nacional de Unidades de Conservação da Natureza e dá outras providências. Disponível em: <http://www.mma.gov.br/port/conama/ legiabre.cfm?codlegi=322>. Acesso em: 03 mar. 2016.

Brasil. Decreto $\mathrm{n}^{\circ} \mathbf{4 . 3 4 0}$, de 22 de agosto de 2002. Regulamenta artigos da Lei $n^{\circ} 9.985$, de 18 de julho de 2000, e dispõe sobre o Sistema Nacional de Unidades de Conservação da Natureza - SNUC, e dá outras providências. Disponível em: <http://www.mma.gov.br/port/ conama/legiabre.cfm?codlegi=374>. Acesso em: 03 mar. 2016.

Brasil. Decreto $\mathbf{n}^{\mathbf{0}}$ 5.566, de 26 de outubro de 2005. Dá nova redação ao caput do art. 31 do Decreto $n^{\circ} 4.340$, de 22 de agosto de 2002, que regulamenta artigos da Lei $\mathrm{n}^{\circ} 9.985$, de 18 de julho de 2000, que dispõe sobre o Sistema Nacional de Unidades de Conservação da Natureza - SNUC. Disponível em: $<$ http://www.planalto.gov.br/ccivil_03/_Ato200 4-2006/2005/Decreto/D5566.htm>. Acesso em: 03 mar. 2016.
Brasil. Decreto $\mathrm{n}^{\mathbf{0}} \mathbf{6 . 8 4 8}$, de 14 de maio de 2009. Altera e acrescenta dispositivos ao Decreto $n^{\circ} 4.340$, de 22 de agosto de 2002, para regulamentar a compensação ambiental. $<$ http://www.planalto.gov.br/ccivil_03/_Ato200 7-2010/2009/Decreto/D6848.htm>. Acesso em: 03 mar. 2016.

Brasil. Resolução CONAMA no ${ }^{0}$, de 03 de dezembro de 1987. Disponível em: <http://www.mma.gov.br/port/conama/legiabre. cfm?codlegi=61>. Acesso em: 03 mar. 2016.

Brasil. Resolução CONAMA $n^{\circ}$ 2, de 18 de abril de 1996. Disponível em: <http://www.mma.gov.br/port/conama/res/res96 /res0296.html>. Acesso em: 03 mar. 2016.

Brasil. Resolução CONAMA n' ${ }^{0}$ 371, de 05 de abril de 2006. Estabelece diretrizes aos órgãos ambientais para o cálculo, cobrança, aplicação, aprovação e controle de gastos de recursos advindos de compensação ambiental, conforme a Lei ${ }^{\circ} 9.985$ de 18 de julho de 2000, que institui o Sistema Nacional de Unidades de Conservação da Natureza - SNUC e dá outras providências. Disponível em: <http://www.mma.gov.br/port/conama/legiabre. cfm?codlegi=493>. Acesso em: 03 mar. 2016.

Millennium Ecosystem Assessment. Ecosystems and human well-being: synthesis. Washington, DC: Island Press, 2005. Disponível em: $\quad<$ http://www.millenniumassessment.org/ documents/document.356.aspx.pdf $>$. Acesso em: 03 mar. 2016.

Motta, R. S. Manual para valoração econômica dos recursos ambientais. Rio de Janeiro: IPEA/MMA/PNUD/CNPq, 1997.

STF - Supremo Tribunal Federal. Ação Direta de Inconstitucionalidade (Med. Liminar) $\mathbf{n}^{\circ}$ 3378, 16 de dezembro de 2004. Disponível em: $<$ http://www.stf.jus.br/portal/peticaoInicial/verP eticaoInicial.asp?base=ADIN\&s1=3378\&proces so=3378> . Acesso em: 03 mar. 2016.

Informação da Licença: Este é um artigo Open Access distribuído sob os termos da Licença Creative Commons Attribution, que permite uso irrestrito, distribuição e reprodução em qualquer meio, desde que a obra original seja devidamente citada. 